\title{
Mixed experimental and numerical approach to evaluation of material parameters of clayey soils
}

\author{
T. Janda, P. Kuklík and M. Šejnoha \\ Czech Technical University in Prague, Faculty of Civil Engineering, \\ Department of Structural Mechanics \\ Thákurova 7, 16629 Prague 6 \\ phone: +420-2-24354494, fax: +420-2-24310775 \\ E-mail: sejnom@fsv.cvut.cz
}

\section{Abstract}

The present contribution reports on a mixed numerical experimental study of the time dependent processes in saturated soils with reference to isotropic consolidation. Owing to its relative simplicity, the isotropic consolidation is often viewed as a basic tool for inferring the material parameters of various constitutive models. Within this modeling framework, the paper advocates the use of the CAM clay model for an accurate description of the time dependent deformation of clayey soils. Apart from the classical formulation based on the normal consolidation line (NCL), the present approach admits two principal modifications. First, both numerical simulations and experimental observations suggest time dependent variation of the permeability coefficient. When expressed as a function of the actual void ratio it can significantly improve the material response particularly when the degree of consolidation increases. To further improve an initial rate of consolidation it appears inevitable to incorporate a time change of the influence zones inside the sample. In the present formulation this is accomplished through a bilinear form of the consolidation line (BCL). Several numerical and experimental results are presented to support the theoretical grounds.

Keywords: Cam Clay, coefficient of permeability, consolidation, BCL, void ratio, structural strength.

\section{Introduction}

Reliable and accurate prediction of the mechanical response of any material including soils depends equally on both the selected constitutive model and material parameters of a given soil entering the model. While the respective constitutive model is usually well defined and tested for the description of a certain type of soils, the values of associated material parameters are mostly lacking that leads to extensive laboratory program for their determination. In certain occasions, however, 
this problem can be confined to a single laboratory test combined with numerical simulations. In particular, the present contribution advocates the use of a simple isotropic consolidation test to infer the basic material parameters describing the modified Cam Clay model.

The modified Cam Clay model has been often a choice of the constitutive model for a realistic representation of the inelastic behavior of clayey soils particularly when deformation of the solid phase is of the main concern. In our previous work, $[8,9]$, we pointed out several drawbacks of this model when applied to consolidation. We recall, in passing, the need for at least bilinear form of the consolidation line (BCL) to account for prior loading history (unloading from a certain level of preconsolidation pressure prior to subsequent loading). The second history requires deformation dependent formulation of the coefficient of permeability, see also [4, 11] and Section 4 for further discussion. Such modifications then give rise to five basic material parameters to feed the constitutive model. These parameters are summarized in Section 2, which briefly reviews the theoretical formulation of isotropic consolidation.

The choice of isotropic consolidation test as a tool for extracting the material parameters of the Cam Clay model is primarily attributed to its relative simplicity and the possibility of the experimental procedure to be exactly reproduced by numerical simulation. Following up the laboratory measurements performed in the triaxial apparatus, the numerical simulation reduces to one-dimensional consolidation problem under fully saturated conditions. Several numerical techniques applicable for the solution of such a problem are discussed in Section 3. Efficiency, accuracy and ease of implementation are the main promoters for their implementation as a part of the optimization problem discussed in Section 5. This section outlines a reliable optimization procedure based on genetic algorithms, [13, 10, 12], to provide optimal values of the desired material parameters by matching experimental measurements with the results derived numerically.

\section{Governing equations}

Referring to experimental measurements carried out in the triaxial apparatus [6], the isotropic consolidation can be viewed as one phase flow in fully saturated deforming medium undergoing small deformation. Geometrical arrangement and loading conditions (cylindrical sample subjected to hydrostatic pressure) allow to write the Cauchy equations of equilibrium in the form

$$
\begin{aligned}
& \frac{\partial \sigma_{x}(x, y, z, t)}{\partial x}=0, \\
& \frac{\partial \sigma_{y}(x, y, z, t)}{\partial y}=0, \\
& \frac{\partial \sigma_{z}(x, y, z, t)}{\partial z}=0 .
\end{aligned}
$$

The hydrostatic state of stress maintained during the experimental measurement together with Eq. (1) give

$$
\sigma_{x}(x, y, z, t)=\sigma_{y}(x, y, z, t)=\sigma_{z}(x, y, z, t)=\frac{\sigma_{m}(t)}{3},
$$




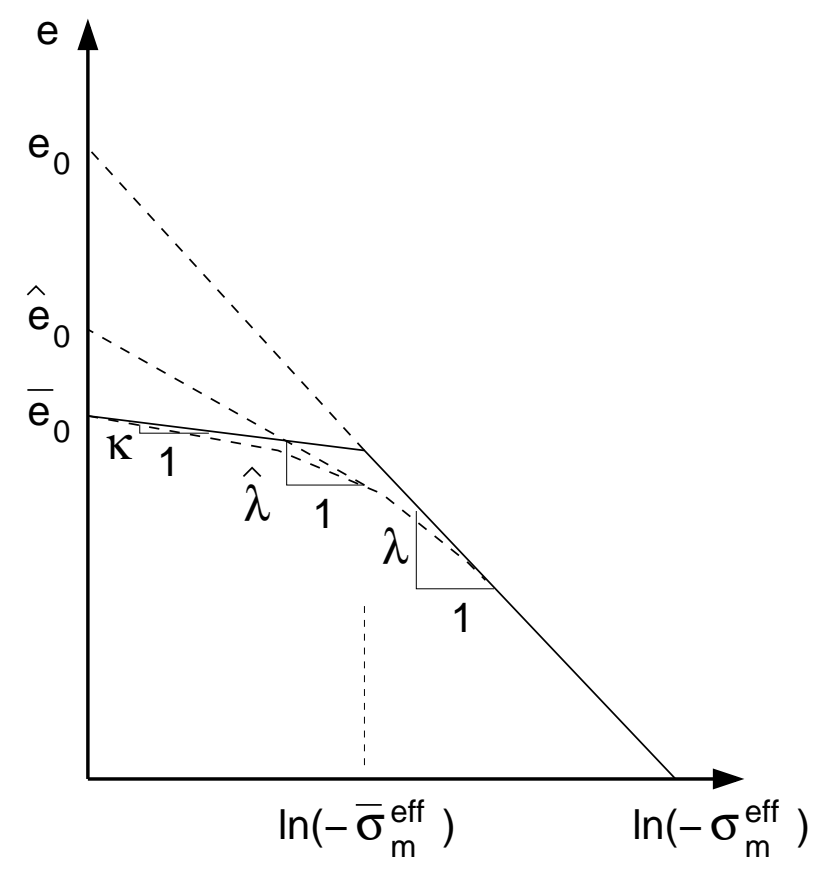

Figure 1: Bilinear form of the consolidation line

where $\sigma_{m}$ is the total mean stress. Following the Terzaghi-Fillunger concept of effective stresses this quantity can be expressed in terms of the pore pressure $p^{s}$ and the effective stresses between grains $\sigma_{m}^{e f f}$ as

$$
\sigma_{m}=\sigma_{m}^{e f f}-p^{s} .
$$

Assuming full saturation $\left(S_{w}=1,[7]\right)$ the pore pressure $p^{s}$ equals the pressure in the liquid phase $p^{w}$. Referring to experimental conditions the total mean stress remains constant throughout the consolidation process. Eqs. (1)-(3) then provide

$$
\dot{\sigma}_{m}=\dot{\sigma}_{m}^{e f f}-\dot{p}^{w}=0,
$$

where $($ ) represents the time derivative $\partial() / \partial t$.

Transport of the liquid phase throughout the soil sample can be described by the following set of equations:

Transport equation

$$
J^{w}=-\frac{K \rho^{w}}{\gamma^{w}} \operatorname{grad} p^{w},
$$

where $J^{w}$ is the mass flux of pore water, $\gamma^{w}=g \rho^{w}$ is the specific weight of water, $\rho^{w}$ is the intrinsic mass density and $K$ represents an instantaneous coefficient of permeability.

Balance equation reads

$$
\rho^{w} \dot{\epsilon}_{v}+\operatorname{div} J^{w}=0 .
$$

The volumetric strain $\epsilon_{v}$ follows from the

Constitutive equation

$$
\begin{array}{rlrl}
\epsilon_{v}=\frac{e-\bar{e}_{0}}{1+\bar{e}_{0}}=-\frac{\kappa}{1+\bar{e}_{0}} \ln \left(-\sigma_{m}^{\text {eff }}\right), & & \sigma_{m}^{\text {eff }}>\bar{\sigma}_{m}^{\text {eff }}, \\
\epsilon_{v}=\frac{e-e_{0}}{1+e_{0}}=-\frac{\lambda}{1+e_{0}} \ln \left(-\sigma_{m}^{\text {eff }}\right), & \sigma_{m}^{\text {eff }}<\bar{\sigma}_{m}^{\text {eff }},
\end{array}
$$


derived in case of the Modified Cam Clay model from the bilinear consolidation line, Fig. 1. The initial branch, often referred to as $\kappa$-line, gives evidence of the previous stress history and represents the effect of overconsolidation. The slope discontinuity between the $\kappa$ and $\lambda$ - lines can be identified with the structural strength of soil given in terms of a certain level of the effective mean stress $\bar{\sigma}_{m}^{\text {eff }}$. The bilinear line displayed in Fig. 1 is usually replaced by a transition along a smooth curve that is characteristic for a given soil under consideration, see the dashed line in Fig. 1. Introducing an instantaneous modulus $\hat{\lambda}$ renders the constitutive equation (7) at an instant time $t$

$$
\epsilon_{v}(t)=\frac{e(t)-\widehat{e}_{0}(t)}{1+\widehat{e}_{0}(t)}=-\frac{\widehat{\lambda}(t)}{1+\widehat{e}_{0}(t)} \ln \left(-\sigma_{m}^{e f f}(t)\right) .
$$

Differentiating Eqs. (8) with respect to time gives the rate of volumetric strain in the form

$$
\dot{\epsilon}_{v}(t)=\frac{\dot{e}(t)}{1+\widehat{e}_{0}(t)}=-\frac{\widehat{\lambda}(t)}{1+\widehat{e}_{0}(t)} \frac{\dot{p}^{w}(t)}{\sigma_{m}^{e f f}(t)} .
$$

Substituting Eqs. (5) and (9) into Eq. (6) and taking into account the actual triaxial set-up, in which only the bottom face of the cylinder is drained, lead to

$$
-\frac{1+\widehat{e}_{0}(t)}{\gamma^{w} \widehat{\lambda}(t)} \sigma_{m}^{e f f}(t) \frac{\partial}{\partial z}\left(K(t) \frac{\partial p^{w}(t)}{\partial z}\right)-\dot{p}^{w}(t)=0 .
$$

It has been verified experimentally that in case of isotropic consolidation a mere power law written as [9]

$$
\frac{K}{K_{0}}=\left(\frac{e}{e_{0}}\right)^{m}
$$

represents the soil behavior fairly well. The dependence of the actual void ratio $e$ on the effective mean stress, Eq. (8), together with Eq. (11) provide

$$
\frac{\partial K(t)}{\partial z}=-\frac{m K(t) \hat{\lambda}(t)}{e(t) \sigma_{m}^{e f f}(t)} \frac{\partial p^{w}(t)}{\partial z}
$$

Introducing Eq. (12) into Eq. (10) finally yields

$$
\dot{p}^{w}(t)=-\frac{K(t)\left(1+\widehat{e}_{0}(t)\right)}{\gamma^{w} \hat{\lambda}(t)}\left[-\frac{m \hat{\lambda}(t)}{e(t)}\left(\frac{\partial p^{w}(t)}{\partial z}\right)^{2}+\sigma_{m}^{e f f}(t) \frac{\partial^{2} p^{w}(t)}{\partial z^{2}}\right] .
$$

\section{Numerical implementation}

The purpose of this section is to evaluate several numerical methods commonly used when solving the consolidation problem. In view of the solution of inverse problem outlined in Section 5, the main objective remains computational efficiency of individual numerical techniques. First, we recall the collocation method successfully implemented in $[8,9]$. The implicit finite control volume scheme combined with the finite difference method is explored next. Finally, our attention is given to the finite element method, which proved to be an efficient tool particularly when taking the volume changes of the porous skeleton into account [2]. 


\subsection{Numerical solution using the collocation method}

A suitable method for solving Eq. (13) combines the collocation method along with a cubic spline approximation of the pore pressure distribution. After discretization Eq. (13) becomes

$$
\dot{p}_{i}^{w}\left(t_{j+1}\right)=-\frac{K\left(t_{j}\right)\left(1+\widehat{e}_{0}\left(t_{j}\right)\right)}{\gamma^{w} \hat{\lambda}\left(t_{j}\right)}\left[-\frac{m \hat{\lambda}\left(t_{j}\right)}{e\left(t_{j}\right)}\left(Y_{i}\left(t_{j}\right)\right)^{2}+\sigma_{m, i}^{e f f}\left(t_{j}\right) M_{i}\left(t_{j+1}\right)\right] .
$$

Note that by using the above approximation we force Eq. (13) to be fulfilled only at a certain points of collocation $(i) ; Y_{i}\left(t_{j}\right)$ and $M_{i}\left(t_{j+1}\right)$ then represent the first and second derivatives of the unknown pore pressure at the $i^{t h}$ point, respectively. Also point out that the pore pressure dependent material parameters $K, \widehat{\lambda}, \widehat{e}_{0}$ and $e$ are taken at time $t=t_{j}$ to avoid solution of the nonlinear system of equations. The same simplification applies to variables $Y_{i}$ and $\left(\sigma_{m}^{\text {eff }}\right)_{i}$. Accepting such assumptions allows to write the above equation in the form

$$
\dot{p}_{i}^{w}\left(t_{j+1}\right)+\alpha\left(t_{j}\right) M_{i}\left(t_{j+1}\right)=\beta\left(t_{j}\right),
$$

where

$$
\begin{aligned}
\alpha_{i}\left(t_{j}\right) & =\frac{K\left(t_{j}\right)\left(1+\widehat{e}_{0}\left(t_{j}\right)\right)}{\gamma^{w} \hat{\lambda}\left(t_{j}\right)} \sigma_{m, i}^{e f f}\left(t_{j}\right) \\
\beta_{i}\left(t_{j}\right) & =\frac{K\left(t_{j}\right)\left(1+\widehat{e}_{0}\left(t_{j}\right)\right)}{\gamma^{w} \hat{\lambda}\left(t_{j}\right)} \frac{m \widehat{\lambda}\left(t_{j}\right)}{e\left(t_{j}\right)}\left(Y_{i}\left(t_{j}\right)\right)^{2}, \\
\sigma_{m, i}^{e f f}\left(t_{j+1}\right) & =\sigma_{m, i}^{e f f}\left(t_{j+1}\right)+p_{i}^{w}\left(t_{j+1}\right)-p_{i}^{w}\left(t_{j}\right) .
\end{aligned}
$$

To solve Eq. (15) we further assume the generalized trapezoidal rule [5] and write

$$
\begin{aligned}
\dot{p}_{i}^{w}\left(t_{j+1}\right) & =\frac{1}{\tau \Delta t}\left(p_{i}^{w}\left(t_{j+1}\right)-\tilde{p}_{i}^{w}\left(t_{j+1}\right)\right), \\
\tilde{p}_{i}^{w}\left(t_{j+1}\right) & =p_{i}^{w}\left(t_{j}\right)+(1-\tau) \Delta t \dot{p}_{i}^{w}\left(t_{j}\right),
\end{aligned}
$$

where $\tau=\left(t-t_{j}\right) / \Delta t ; \Delta t=t_{j+1}-t_{j}$ is the time step. Parameter $\tau$ should be chosen from $\tau \in(1 / 2,1)$ to ensure numerical stability. The most common choice is $\tau=1 / 2$. When substituting Eqs. (16) and (17) into Eq. (15) we arrive at

$$
p_{i}^{w}\left(t_{j+1}\right)=-\tau \Delta t \alpha_{i}\left(t_{j}\right) M_{i}\left(t_{j+1}\right)+\tau \Delta t \beta_{i}\left(t_{j}\right)+\tilde{p}_{i}^{w}\left(t_{j+1}\right) .
$$

Further suppose an equidistant spread of the collocation points with step $\Delta h=$ $z_{i}-z_{i-1}$. The cubic spline equation then reads

$$
\frac{1}{2} M_{i-1}+2 M_{i}+\frac{1}{2} M_{i}=\frac{3}{\Delta h^{2}}\left(p_{i-1}-2 p_{i}+p_{i+1}\right) .
$$

Combining the Eqs. (19) and (18) then readily provides the final tridiagonal system of algebraic equations in the form

$$
\begin{aligned}
& {\left[\frac{1}{2}+3 \tau \Delta t \alpha_{i-1}\left(t_{j}\right) \frac{1}{\Delta h^{2}}\right] M_{i-1}\left(t_{j+1}\right) } \\
+ & {\left[2-6 \tau \Delta t \alpha_{i}\left(t_{j}\right) \frac{1}{\Delta h^{2}}\right] M_{i}\left(t_{j+1}\right) } \\
+ & {\left[\frac{1}{2}+3 \tau \Delta t \alpha_{i+1}\left(t_{j}\right) \frac{1}{\Delta h^{2}}\right] M_{i+1}\left(t_{j+1}\right) } \\
= & \frac{3}{\Delta h^{2}}\left[P_{i-1}\left(t_{j}\right)-2 P_{i}\left(t_{j}\right)+P_{i+1}\left(t_{j}\right)\right],
\end{aligned}
$$




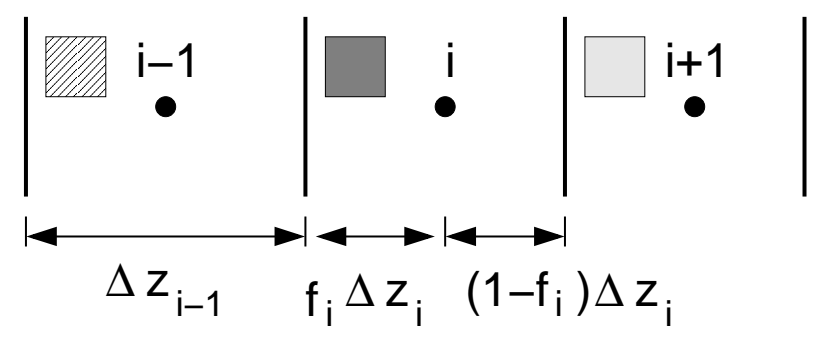

Figure 2: A three layer model

where

$$
\begin{aligned}
P_{i}\left(t_{j}\right) & =\tau \Delta t \beta_{i}\left(t_{j}\right)+\tilde{p}_{i}^{w}\left(t_{j+1}\right), \\
Y_{i}\left(t_{j}\right) & =\frac{1}{\Delta h}\left(p_{i}^{w}\left(t_{j}\right)-p_{i-1}^{w}\left(t_{j}\right)\right)+\frac{\Delta h}{6}\left(2 M_{i}\left(t_{j}\right)+M_{i-1}\left(t_{j}\right)\right) .
\end{aligned}
$$

To complete the numerical procedure the following boundary and initial conditions are supplemented in accord with the experimental set up

$$
\begin{aligned}
p_{1}\left(t_{j}\right) & =0, \quad p_{n}\left(t_{j}\right)=0, \\
p_{i}(0) & =-\sigma_{m}+\sigma_{m, i}^{\text {eff }}(0) \quad i=2, \ldots, n-1,
\end{aligned}
$$

where $n$ is the number of collocation points. The selected cubic spline boundary conditions assume the form

$$
M_{1}\left(t_{j}\right)=M_{n}\left(t_{j}\right)=0
$$

\subsection{Numerical solution using the finite volume method}

It is evident that direct solution of Eq. (13) leads, in general, to a nonlinear system of equations. Therefore it appears preferable to start by discretizing the governing equations, which are then successively used in single time step. In view of onedimensional problem, Eq. (10), the set of governing equations will be discretized using a three-layer model. Individual layers (finite control volumes) have different thickness and diverse materials can be assigned to each layer. The pore pressure is assumed to be constant inside each control volume and within each time step, and to be equal to the pore pressure at the so called grid-point, Fig. 2. Its position is prescribed by a chosen parameter $f_{i}$.

Transfer equation

Application of the finite difference scheme converts Eq. (5) for the water flux throughout a layer $i$ with thickness $\Delta z_{i}$ into the form

$$
J_{i}^{w}=-\frac{K_{i}}{g \Delta z_{i}}\left(p_{i}^{w}-p_{i-1}^{w}\right)=-\left(h z_{i}\right)\left(p_{i}^{w}-p_{i-1}^{w}\right),
$$

where

$$
\frac{1}{h z_{i}}=\left(1-f_{i-1}\right) \frac{g \Delta z_{i-1}}{K_{i-1}}+f_{i} \frac{g \Delta z_{i}}{K_{i}}
$$

is the overall resistance between grid points $(i-1)$ and $i$. 


\section{Balance equation}

Employing the generalized trapezoidal method provides the time variation and the rate of volumetric strain in the form, recall Eqs. (16) and (17),

$$
\begin{aligned}
& \dot{\epsilon}_{v, i}\left(t_{j+1}\right)=\frac{1}{\tau \Delta t}\left(\epsilon_{v, i}\left(t_{j+1}\right)-\tilde{\epsilon}_{v, i}\left(t_{j+1}\right)\right), \\
& \tilde{\epsilon}_{v, i}\left(t_{j+1}\right)=\epsilon_{v, i}\left(t_{j}\right)+(1-\tau) \Delta t \dot{\epsilon}_{v, i}\left(t_{j}\right) .
\end{aligned}
$$

Next, applying again the finite difference scheme yields an incremental form of the balance equation (6) for layer $i$

$$
\frac{\Delta z_{i} \rho^{w}}{\tau \Delta t}\left(\epsilon_{v, i}\left(t_{j+1}\right)-\tilde{\epsilon}_{v, i}\left(t_{j+1}\right)\right)+J_{i+1}^{w}\left(t_{j+1}\right)-J_{i}^{w}\left(t_{j+1}\right)=0
$$

State equation

Introducing Eqs. (16) and (27) into Eq. (9) gives

$$
\epsilon_{v, i}\left(t_{j+1}\right)-\tilde{\epsilon}_{v, i}\left(t_{j+1}\right)=-\frac{\hat{\lambda}\left(t_{j}\right)}{1+\widehat{e}_{0}\left(t_{j}\right)} \frac{1}{\sigma_{m, i}^{e f f}\left(t_{j}\right)}\left(p_{i}^{w}\left(t_{j+1}\right)-\tilde{p}_{i}^{w}\left(t_{j+1}\right)\right) .
$$

After combining Eqs. (30), (29) and (25) we arrive at a set of tridiagonal algebraic equations in the form

$$
\begin{aligned}
& \left(h z_{i+1}\right) p_{i+1}^{w}\left(t_{j+1}\right)-\left[\left(h z_{i+1}\right)+\left(h z_{i}\right)-\frac{\Delta z_{i} \rho^{w}}{\tau \Delta t} \frac{\widehat{\lambda}\left(t_{j}\right)}{1+\widehat{e}_{0}\left(t_{j}\right)} \frac{1}{\sigma_{m, i}^{e f f}\left(t_{j}\right)}\right] p_{i}^{w}\left(t_{j+1}\right) \\
& +\left(h z_{i}\right) p_{i-1}^{w}\left(t_{j+1}\right)=\frac{\Delta z_{i} \rho^{w}}{\tau \Delta t} \frac{\widehat{\lambda}\left(t_{j}\right)}{1+\widehat{e}_{0}\left(t_{j}\right)} \frac{1}{\sigma_{m, i}^{e f f}\left(t_{j}\right)} \tilde{p}_{i}^{w}\left(t_{j+1}\right)
\end{aligned}
$$

At the onset of consolidation the initial condition, similar to Eq. (23), reads

$$
p_{i}(0)=-\sigma_{m}+\sigma_{m, i}^{e f f}(0) \quad i=1, \ldots, n,
$$

where $n$ is the number of finite control volumes.

\subsection{Numerical solution using the finite element method}

In view of the general solution of fluid-solid interaction we recall the finite element method (FEM) often used to solve differential equations similar to Eq. (10). Apart from Eq. (10), which must be satisfied at any point inside the soil body, the boundary value problem requires formulation of the boundary conditions. To that end, the boundary is decomposed into two parts, $\Gamma=\Gamma_{p}+\Gamma_{q}$, with the following boundary conditions:

Essential boundary condition

$$
p^{w}=\bar{p}^{w}, \quad \text { on } \Gamma_{p}
$$

Natural boundary condition

$$
\frac{1+\widehat{e}_{0}}{\widehat{\lambda}} \frac{\sigma_{m}^{e f f}}{\gamma_{w}} K \frac{\partial p}{\partial z}+\bar{q}=0, \quad \text { on } \Gamma_{q}
$$




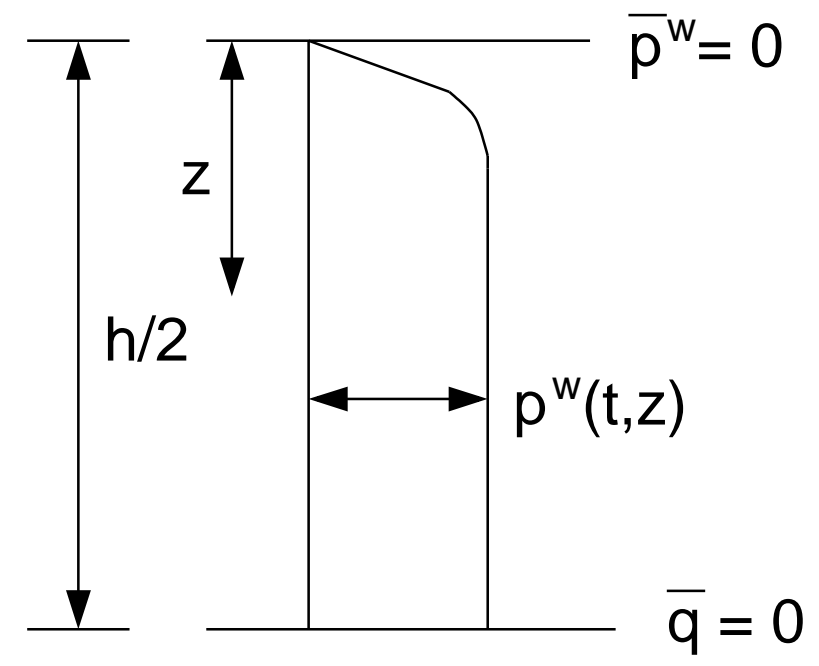

Figure 3: Boundary conditions

Fig. 3 shows the boundary conditions pertinent to the isotropic consolidation.

To derive a set of finite element equations we start by introducing the balance equation (10) and its natural boundary condition (34) into the principle of virtual work written as

$$
\begin{aligned}
& \int_{0}^{H}\left[-\frac{1+\widehat{e}_{0}}{\gamma^{w} \widehat{\lambda}} \sigma_{m}^{e f f} \frac{\partial}{\partial z}\left(K \frac{\partial p^{w}}{\partial z}\right)-\dot{p}^{w}\right] \delta p^{w} \mathrm{~d} z \\
& +\left[\frac{1+\widehat{e}_{0}}{\widehat{\lambda}} \frac{\sigma_{m}^{e f f}}{\gamma_{w}} K \frac{\partial p}{\partial z}+\bar{q}\right]_{z=\frac{H}{2}} \delta p^{w}(H)=0 .
\end{aligned}
$$

Integrating the first term in Eq. (35) by parts yields

$$
\int_{0}^{H} \frac{\partial \delta p^{w}}{\partial z} \frac{1+\widehat{e}_{0}}{\gamma^{w} \hat{\lambda}} K \sigma_{m}^{e f f} \frac{\partial p^{w}}{\partial z} \mathrm{~d} z-\int_{0}^{H} \delta p^{w} \dot{p}^{w} \mathrm{~d} z+\bar{q} \delta p^{w}(H)=0 .
$$

The next step employs the usual approximation of the water pore pressure in the form

$$
p^{w}=\mathbf{N}_{p} \boldsymbol{d}_{p}
$$

where $\mathbf{N}_{p}$ stores the element shape functions and $\boldsymbol{d}_{p}$ is the vector of nodal pore water pressures. After substituting the above approximation into Eq. (36) we identify the following matrices, [7],

permeability matrix

$$
\mathbf{P}=\int_{0}^{H} \frac{1+\widehat{e}_{0}}{\gamma^{w} \hat{\lambda}} K \sigma_{m}^{e f f}\left(\mathbf{N}_{p}^{\prime}\right)^{\mathrm{T}} \mathbf{N}_{p}^{\prime} \mathrm{d} z, \quad N_{k, p}^{\prime}=\frac{\partial N_{k, p}}{\partial z},
$$

compressibility matrix

$$
\mathbf{C}=-\int_{0}^{H} \mathbf{N}_{p}^{\mathrm{T}} \mathbf{N}_{p} \mathrm{~d} z .
$$

Note that the last term in Eq. (36) drops out due to prescribed boundary conditions. Since Eq. (36) must be satisfied for any admissible virtual pore pressure $\delta p^{w}$ we arrive at the following system of equations

$$
\mathbf{C} \dot{\boldsymbol{d}}_{p}\left(t_{j+1}\right)+\mathbf{P}\left(t_{j}\right) \boldsymbol{d}_{p}\left(t_{j+1}\right)=\mathbf{0} .
$$


We usually require the material parameters, including the coefficient of permeability, be constant within a given element. Eqs. (7) and (11) suggest the volumetric strain to be constant as well. When assuming a linear approximation of the pore pressure, the vector of nodal pore pressure values of the $i^{\text {th }}$ element becomes

$$
\left(\boldsymbol{d}_{i, p}\right)^{\mathrm{T}}=\left\{d_{p 1}, d_{p 2}\right\}_{i} .
$$

The constant effective mean stress $\sigma_{i, m}^{e f f}$ in the $i^{\text {th }}$ element is then given by

$$
\sigma_{i, m}^{e f f}=\sigma_{m}-\frac{\left(d_{p 1}+d_{p 2}\right)_{i}}{2}
$$

For the sake of consistency, the volumetric strain $\epsilon_{v}$ and the water pore pressure $p^{w}$ should be of the same order (recall Eq. (9). Herein, the desirable consistency of the solution is achieved by applying the so-called selective integration to integrate the compressibility matrix (one-point Gauss' quadrature).

Once the discretization in space has been performed, Eq. (40) represents a set of ordinary differential equations in time, which can be integrated numerically, [2]. To proceed, consider again the generalized trapezoidal rule such that

$$
\begin{aligned}
& \dot{\boldsymbol{d}}_{p}\left(t_{j+1}\right)=\frac{1}{\tau \Delta t}\left(\boldsymbol{d}_{p}\left(t_{j+1}\right)-\tilde{\boldsymbol{d}}_{p}\left(t_{j+1}\right)\right), \\
& \tilde{\boldsymbol{d}}_{p}\left(t_{j+1}\right)=\boldsymbol{d}_{p}\left(t_{j}\right)+(1-\tau) \Delta t \dot{\boldsymbol{d}}_{p}\left(t_{j}\right) .
\end{aligned}
$$

Substitution of Eq. (43) into Eq. (40) finally leads to a system of linear algebraic equations given by

$$
\frac{1}{\tau \Delta t}\left(\mathbf{C}+\tau \Delta t \mathbf{P}\left(t_{j}\right)\right) \boldsymbol{d}_{p}\left(t_{j+1}\right)=\frac{1}{\tau \Delta t} \mathbf{C} \tilde{\boldsymbol{d}}_{p}\left(t_{j+1}\right) .
$$

The initial condition prescribing the water pore pressure at the beginning of loading, $t=0$ takes the form

$$
\boldsymbol{d}_{i, p}=\boldsymbol{d}_{i, p}(0)=-\sigma_{m}+\sigma_{i, m}^{e f f}(0) \quad i=1, \ldots, n,
$$

where $n$ is the number of elements.

\section{Numerical results}

Before proceeding with the results found using the presented formulation we draw the readers attention to Fig. 4 manifesting several drawbacks associated with the solution of the consolidation problem when applied to the standard Cam Clay model. The solid line shows results derived experimentally. The dash-dotted line follows from numerical calculations assuming the liner NCL (virgin soil) and the deformation dependent coefficient of permeability, while the dashed line was found from the BCL but keeping the coefficient of permeability constant. Clearly, neither the deformation dependent variation of the coefficient of permeability nor the bilinear form of the consolidation line can itself describe the true consolidation process and therefore both of them should be taken into consideration when simulating transport of water in soft soils. The material parameters entering Eq. (13) are listed in Table 1. 


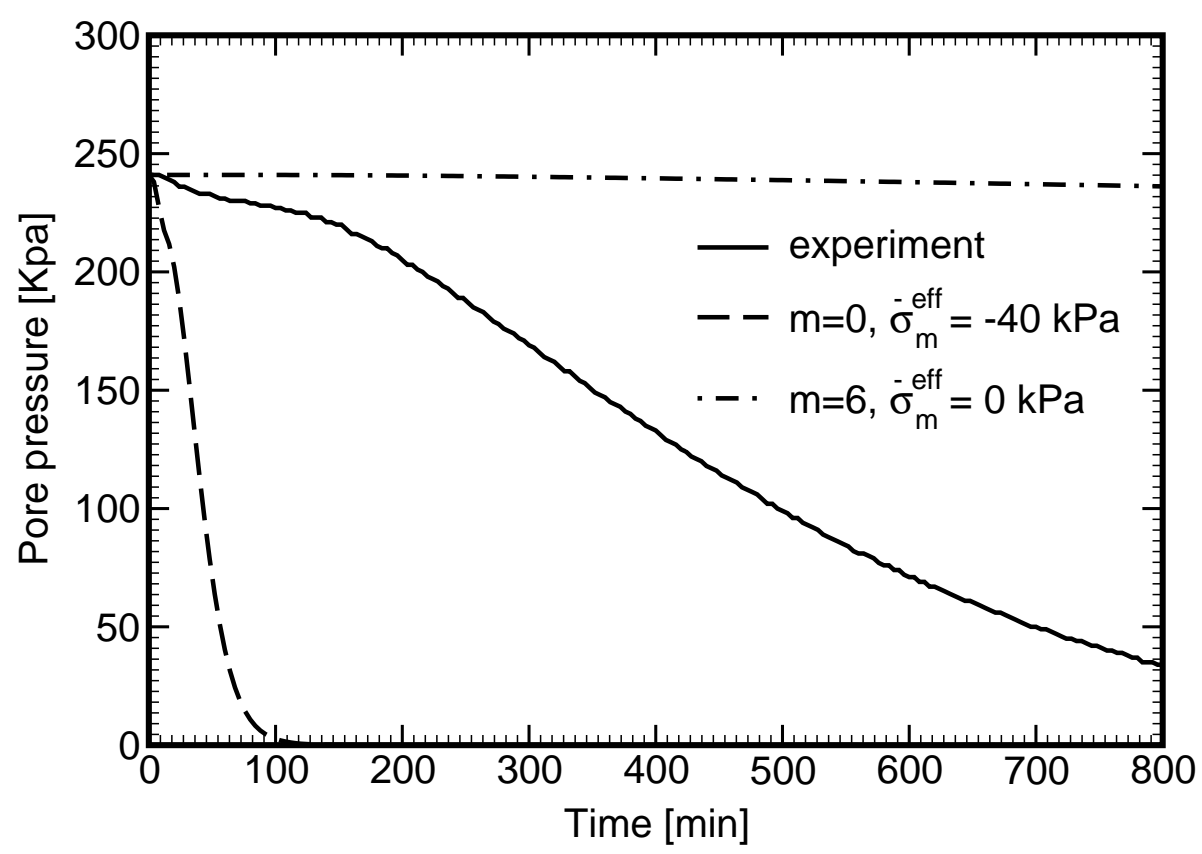

Figure 4: Time variation of pore pressure

Table 1: Material parameters

\begin{tabular}{cccccc}
\hline$\kappa[\mathrm{kPa}]$ & $\lambda[\mathrm{kPa}]$ & $e_{0}$ & $K_{0}$ & $\bar{\sigma}_{m}^{\text {eff }}[\mathrm{kPa}]$ & $m$ \\
\hline 0.01 & 0.1 & 0.5 & $5 \mathrm{e}-9$ & -40 & 6 \\
\hline
\end{tabular}

In a nutshell, the first assumption essentially labels soils as being normally consolidated, which further contributes to a rather slow initial phase of the process of consolidation, Fig. 4 (dash-dotted line). In natural state, however, soils are usually in overconsolidated states due to unloading from the original stress state (e.g., water table fluctuation). To account for a prior loading history we introduce the influence zone variation as a function of a certain level of the effective mean stress, usually referred to as the structural strength. This level of the effective mean stress can be identified with the preconsolidation pressure. In the present formulation, the structural strength is introduced trough a bilinear shape of the consolidation line [9], [1]. An effect of this modification becomes evident when examining Eq. (13). In particular, a lower value of $\hat{\lambda}$ at the initial stage increases the coefficient of permeability leading to an acceleration of the consolidation process at this stage, a phenomenon observed in experiments.

The second shortcoming comes from the originally assumed constant value of the coefficient of permeability, responsible for an abrupt increase in rate of consolidation as the effective mean stress developed during consolidation becomes appreciable, Fig. 4 (dashed line). However, this stage of consolidation is associated with a significant deformation of the skeleton leading to the decrease of the volume of pores and subsequently to the decrease of the coefficient of permeability. Based on our extensive numerical investigations of the isotropic consolidation [8], [9] we proposed an exponential relation between the coefficient of permeability and the actual void ratio 


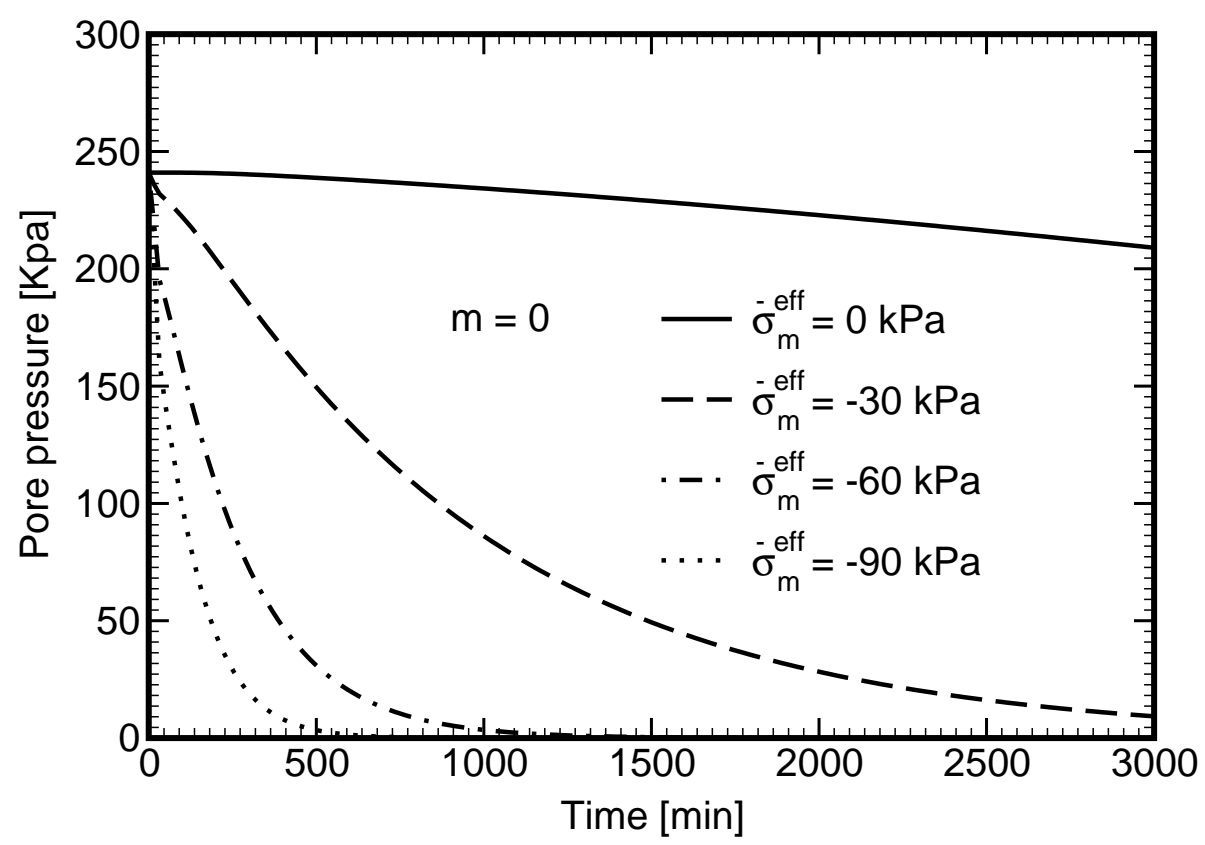

Figure 5: Time variation of pore pressure

represented by Eq. (11). Note that this relationship was suggested by the authors based solely on numerical results. Nevertheless, it conforms with an experimental observation, [11] pp. 138, fairly well.

The remaining part of this section provides qualitative description of individual modifications. First, an influence of the BCL, Fig. 1, is investigated. The location of the slope discontinuity on the BCL corresponds to a given value of the structural strength of soil. This value can be estimated using, e.g., the Casagrande method [3]. To avoid numerical difficulties around this point we equipped the diagram with a cubic parabola to smooth out the transition zone. Results for various values of the structural strength $\bar{\sigma}_{m}^{\text {eff }}$ are plotted in Fig. 5 assuming $m$ equal to 6 . It is evident that increasing the magnitude of $\bar{\sigma}_{m}^{e f f}$ speeds up the rate of consolidation in the first stage quite substantially.

The second modification aims at slowing down the consolidation process in the second stage of consolidation associated with decreasing volume of pores due to skeleton deformation. This phenomenon is taken into account by incorporating Eq. (11) into numerical procedure. Results for various values of exponent $m$ and for a given structural strength $\bar{\sigma}_{s}^{e f f}=-30 \mathrm{kPa}$ are presented in Fig. 6. The dash-dotted line shows rather gradual decrease of the pore pressure profile with increasing time which is in better agreement with experimental observations.

Fig. 7 then summarizes the present theory. First, recall that the dotted line was obtained without taking into account the first modification. In such a case a rather high value of $m$ locks up the numerical procedure at the beginning of consolidation. The dashed line then reflects suggested modifications. At the present time a simple trial and error procedure was employed to derive optimal values of $m$ and $\bar{\sigma}_{m}^{\text {eff }}$. However, a simple least square based optimization procedure can be inferred to obtain better match between experiment and numerical simulation. This problem is addressed in Section 5. 


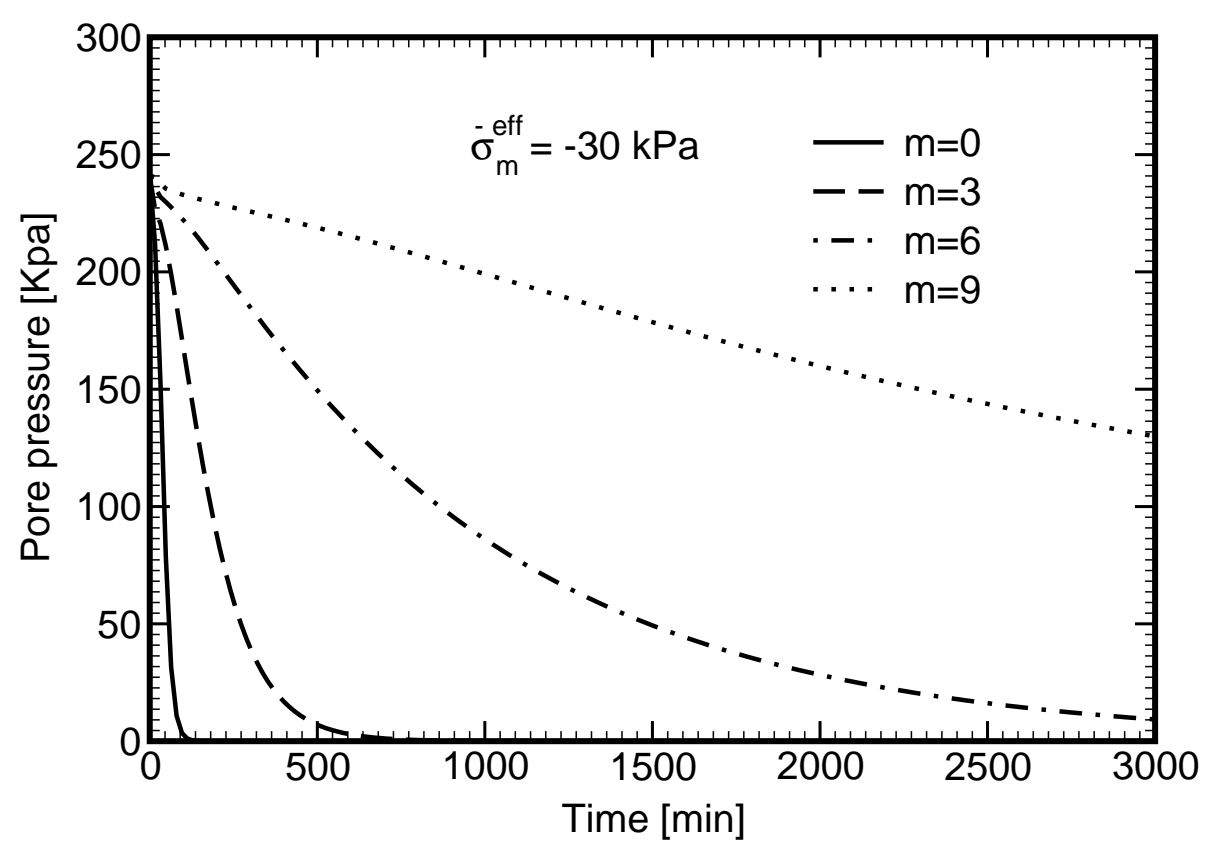

Figure 6: Time variation of pore pressure

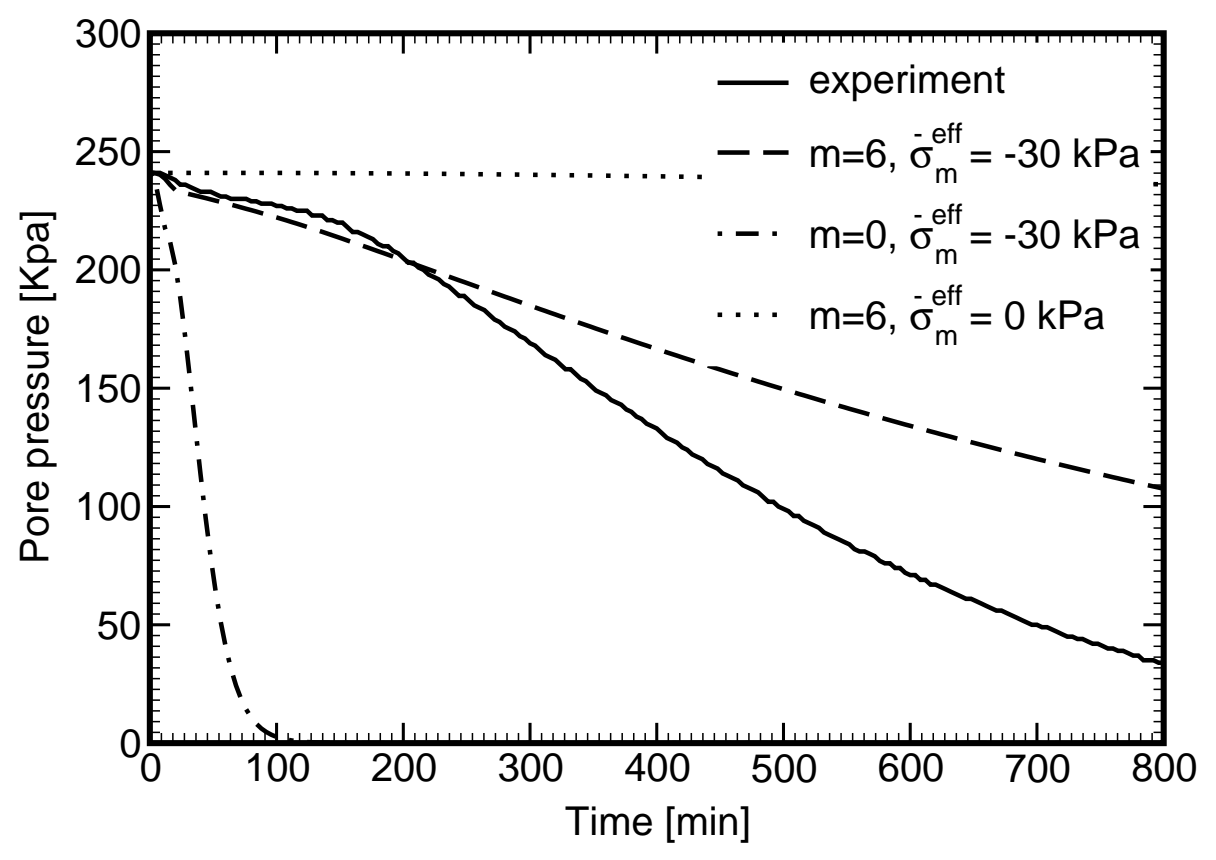

Figure 7: Time variation of pore pressure 


\section{Formulation of the optimization problem}

As intimated in the introductory section, the one-dimensional isotropic consolidation can be used very efficiently to extract basic material parameters of the modified Cam Clay model from a single laboratory test. Matching experimentally obtained data with those derived numerically it might be the simplest view at a complex optimization procedure discussed herein.

Recall Eq. (13) describing the excess pore pressure variation during consolidation. Reproducing laboratory data thus requires to supply the following material parameters: the initial void ratio $\bar{e}_{0}$, the swelling index $\kappa$, the compression index $\lambda$, the structural strength parameter $\bar{\sigma}_{m}^{e f f}$ and the parameter $m$ in Eq. (11). In view of the optimization problem these parameters now become the search variables to be found by minimizing the following function

$$
F=\sum_{k=1}^{K}\left(p^{w}\left(t_{k}\right)-\bar{p}^{w}\left(t_{k}\right)\right)^{2},
$$

where $\bar{p}^{w}\left(t_{k}\right)$ is an experimentally obtained value of the pore pressure at a certain time instant $t_{k}$ and $K$ is the number of supplied measurements taken at times $t_{k}, k=$ $1,2, \ldots, K$. To facilitate the step-by-step solution of Eq. (13), we first set time integration steps as differences between matching times $t_{k}$ (i.e. $(\Delta t)_{k}=t_{k}-t_{k-1}$ ). Pore pressure values $p^{w}\left(t_{k}\right)$ then follow from Eq. (13).

\subsection{Genetic-algorithm based optimization procedure}

As suggested by the authors the minimization of Eq. (47) can be tackled very efficiently with the help of problem solving systems based on principles of evolution such as genetic algorithms (GAs) or augmented simulated annealing methods [13, 10, among others]. Detailed exposition to a number of genetic algorithms adopted for solving various engineering problems is given in [12].

Based on our experience with genetic algorithms we implemented here a very efficient and reliable optimization technique called the augmented (or parallel) simulated annealing (AUSA) [10]. This method effectively combines the essentials of GAs (a population of possible solutions $P$ termed chromosomes, rather then a single point in space, is optimized) together with the basic concept of simulated annealing method guiding the search towards minimal energy states. The GA-phase closely follows the basic steps of a single GA cycle: reproduction, recombination, and selection of a new population. From this viewpoint we may relate the AUSA to a group of Steady state and On the fly methods [13], in which offspring replace its parents immediately. The following algorithm describes an implementation of the AUSA:

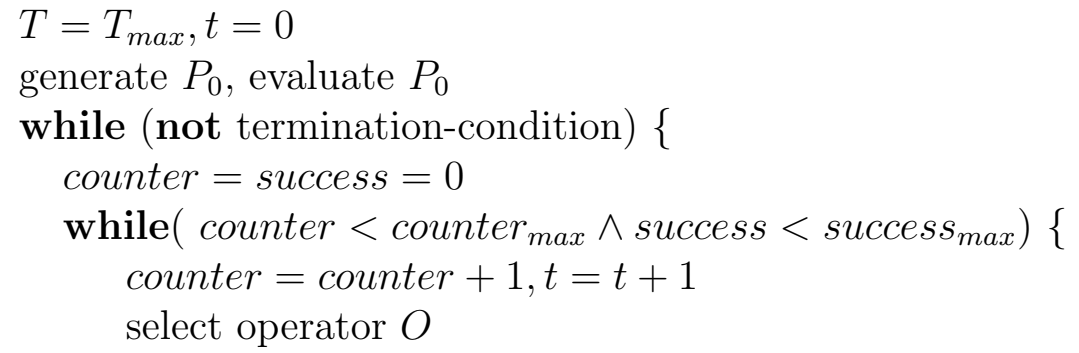




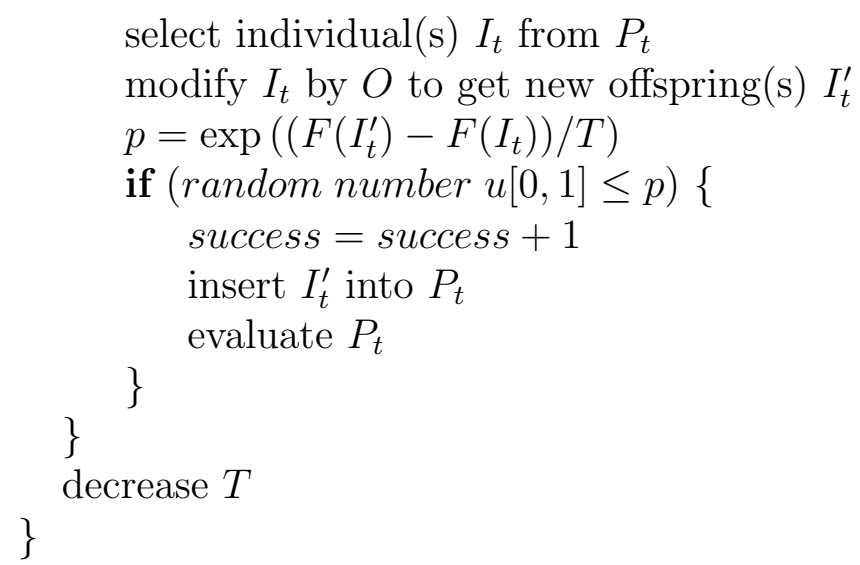

Algorithm 1: Augmented Simulated Annealing

To draw the reader closer to genetic algorithms we now describe the essential features of the above algorithm in conjunction with the present minimization problem. In general, function $F$ in Eq. (47) is a function of 5 variables

$$
F(\boldsymbol{X})=F\left(\bar{e}_{0}, \kappa, \lambda, \bar{\sigma}_{m}^{e f f}, m\right) .
$$

To keep up with the genetic algorithm vocabulary we shall call the vector $\boldsymbol{X}$ a chromosome and individual components genes. Step 2 in Algorithm 1 requires to generate a family of possible solutions called population. To that end, we first estimate a possible solution using either a simple trial and error method (Section 4) or data available for a similar type of soil. Next, setting the upper and lower bounds on individual search variables, we generate individual chromosomes in the initial population purely randomly.

As in nature, where species compete for their survival, the selection of chromosomes for their reproduction, step 8, depends on their current fitness. The fitter ones have better chance to be selected. In the present minimization problem the fitness is simply equal to inverse of the function value

$$
s_{i}=\frac{1}{\delta+F_{j}}, \quad F_{j} \geq 0,
$$

where $F_{j}$ is the function value associated with the $j$ th individual. Parameter $\delta$ is a small positive number. Note that $s_{j}=F_{j}$ when solving maximization problem. Various selection methods are available in [12].

Step 9 requires application of certain genetic operators. Recall that breeding is the essential force driving evolution of each species. Mating process, in which two parents combine their good characteristics to produce a better offspring, is accomplished in genetic algorithms through various "cross-breeding" and "mutating" operators. Due to nature of vector $\boldsymbol{X}$ we choose float point representation of searched variables thus no mapping between search and representation space is required. Detailed exposition to genetic operators built for float point representation is given in $[13,12]$. Here, we limited our attention to nonuniform mutation and simple arithmetic crossover defined in the sequel.

Let $L_{i}$ and $U_{i}$ represent the lower and upper bound for each variable $x_{i}$, respectively. Further assume that vector $\boldsymbol{X}$ represents a parent, whereas vector $\boldsymbol{X}^{\prime}$ 
corresponds to an offspring; $u(a, b)$ is a real number and $u[a, b]$ is an integer number with uniform distribution defined on a closed interval $\langle a ; b\rangle$. The following operators can be now defined:

Non-uniform mutation: Let $j=u[1,2 N], p=u(0,1)$ and set:

$$
x_{i}^{\prime}=\left\{\begin{array}{cl}
x_{i}+\left(L_{i}-x_{i}\right) f(t), & \text { if } i=j, p<.5 \\
x_{i}+\left(U_{i}-x_{i}\right) f(t), & \text { if } i=j, p \geq .5 \\
x_{i}, & \text { otherwise }
\end{array}\right.
$$

where $f(t)=u(0,1)\left(1-t / t_{\max }\right)^{b}, t$ is the current generation, $t_{\max }$ is the maximum number of generations and $b$ is the shape parameter. This operator allows for a local tuning as it searches the space uniformly initially and very locally at later stages.

Simple arithmetic crossover: Let $j=u[1,2 N], p=u(0,1)$ and set:

$$
\begin{aligned}
& x_{i}^{\prime}=\left\{\begin{array}{cc}
p x_{i}+(1-p) y_{i}, & \text { if } i=j \\
x_{i}, & \text { otherwise }
\end{array}\right. \\
& y_{i}^{\prime}=\left\{\begin{array}{cc}
p y_{i}+(1-p) x_{i}, & \text { if } i=j \\
y_{i}, & \text { otherwise }
\end{array}\right.
\end{aligned}
$$

In the present formulation the reproduction step proceeds in two steps. First, we select parents (chromosomes) to breed according to their fitness. Genetic operators, however, are not applied to the entire chromosome but rather to individual genes. It is recommended to choose mutation operators with much higher probabilities then crossovers. In $[10]$ ratio $\approx 0.1$ is proposed.

The replacement procedure (steps 10 through 15) is controlled by the Metropolis criterion, which allows a worse child to replace its better parent with only a certain probability. The probability of excepting a worse solution is reduced as the procedure converges to the "global" minimum. To that end a certain cooling schedule must be created. We use a very simple form of cooling schedule $T_{i+1}=T_{\text {mult }} T_{i}$. In this step we also perform reannealing if necessary. If the actual temperature is lower then a given parameter $T_{\min }$, we set $T=T_{\max }$ and copy a half of the current population to a new one. Remaining part of a new population is generated randomly. The temperature $T_{\max }$ should be chosen such that the ratio of accepted solutions to all solutions is $\approx 50 \%$.

Clearly, evaluation of the objective function calls for a fast numerical procedure to solve Eq. (13) as the number of function evaluation may exceed several hundreds. It has been confirmed that any of the three techniques outlined in Section 3 is equally efficient and therefore no preference is given to any of them. It is further expected that rather accurate estimate of the initial solution may substantially speed up the optimization process.

\section{$5.2 \quad$ Numerical results}

Numerical simulations obtained using the material parameters found from the optimization process are plotted in Fig. 8. The corresponding material properties are 


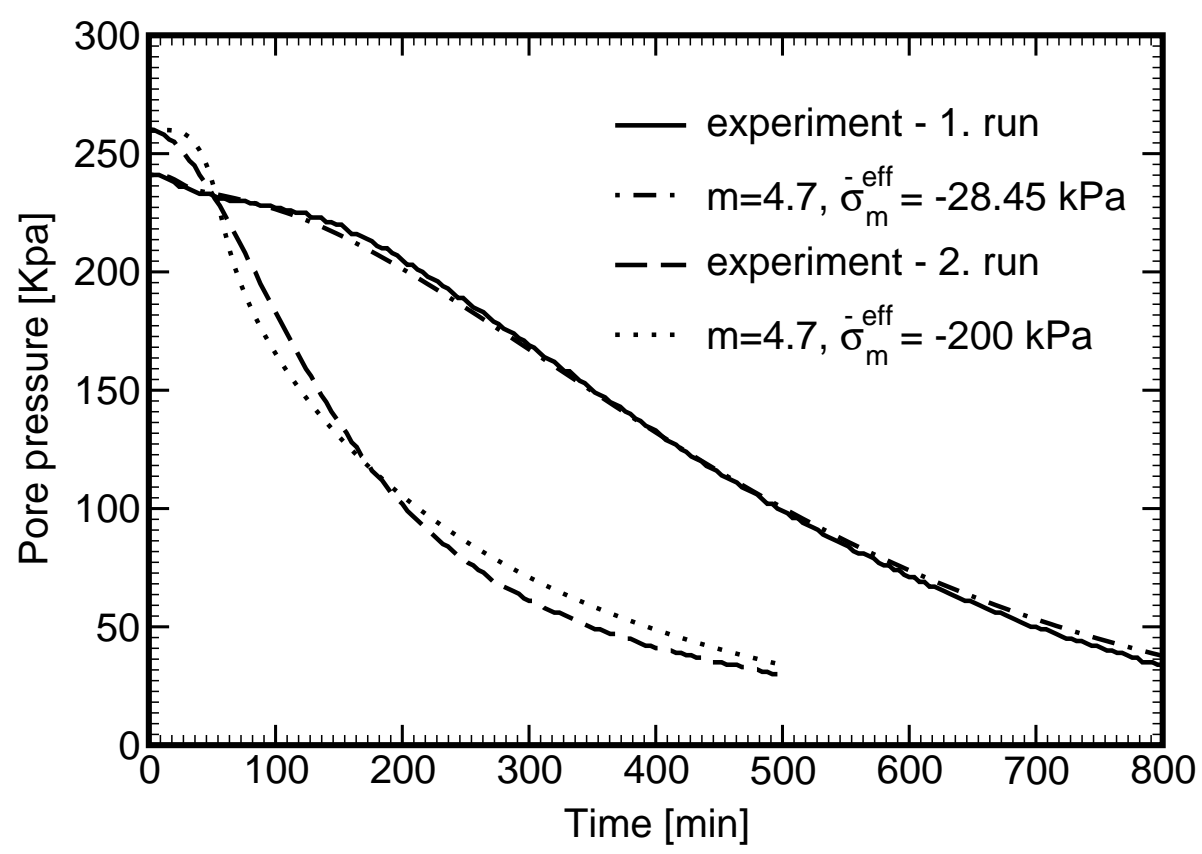

Figure 8: Time variation of pore pressure

Table 2: Optimal material parameters

\begin{tabular}{cccccc}
\hline$\kappa[\mathrm{kPa}]$ & $\lambda[\mathrm{kPa}]$ & $e_{0}$ & $K_{0}$ & $\bar{\sigma}_{m}^{e f f}[\mathrm{kPa}]$ & $m$ \\
\hline 0.012 & 0.074 & 0.56 & $1.53 \mathrm{e}-9$ & -28.45 & 4.7 \\
\hline
\end{tabular}

stored in Table 2. To derive the dash-dotted line we started from material properties provided by Table 1 as our initial guess. Minimizing Eq. (47) then let to the desired material properties describing the true behavior of the selected soil. Perfect match between experimental and numerical data is evident (see the solid and dash-dotted lines in Fig. 8). To confirm the theoretical formulation we rerun the laboratory test on the previously consolidated soil. Note the level of preconsolidation pressure equal to about $-200 \mathrm{kPa}$ resulting from the initial experimental measurements as well as associated numerical calculations. This value was taken as a measure of the structural strength for the numerical simulation in the second run. In addition, the initial coefficient of permeability was set equal to $0.43\left(K_{0}=4.5 \mathrm{e}-9\right)$. This value was found upon unloading at the end of the initial consolidation run using the optimal $\kappa$ modulus (see also Fig. 1). The remaining material data were taken from Table 2. The results appear in Fig. 8. The dashed line corresponds to experimental measurements, whereas the dotted line was constructed numerically. Agreement between laboratory and numerical experiments is remarkable. It is worthwhile to mention a noticeable speedup in the course of consolidation attributed to an increase of the structural strength after terminating the initial consolidation process. This final result further supports the validity of the proposed theoretical formulation. 


\section{Conclusions}

The present paper was concerned with an effect of structural strength in the computational model of isotropic consolidation in which the skeleton deformation is governed by the modified Cam Clay model. Several numerical techniques to solve the isotropic consolidation problem were explored. Both the numerical results and experimental data proved necessity of incorporating the structural strength of soils into the computational model. Experimental measurements verified that soils with a higher magnitude of the structural strength are characterized by a shorter time of consolidation which could not be accounted for by the standard modified Cam Clay model (virgin line of isotropic consolidation). In addition a number of numerical as well experimental results verified the proposed modification of the coefficient of permeability. An independent experimental observation confirmed an exponential form of the relationship between the coefficient of permeability and current void ratio proposed by the authors. Comparison of numerical results with experiments suggest that both modifications are important and are not mutually interchangeable. It was further suggested that isotropic consolidation formulated as an optimization problem can serve as an efficient tool to extract the material parameters describing the modified Cam Clay model. To that end, a reliable optimization technique based on the AUSA was proposed.

\section{Acknowledgments}

Financial support for this project was provided by the Ministry of education, projects No. MSM:210000001 and MSM:210000003.

\section{References}

[1] Asaoka, A., Nakano, M., Noda, T. Super loading yield surface concept for the saturated structured soils, NUMGE98, edited by A. Cividini, Udine, Italy, 1998; 233-242.

[2] Bittnar, Z. and Šejnoha, J. Numerical methods in structural mechanics, ASCE Press, NY, 1996.

[3] Bowles, J.E. Foundation analysis and design, McGraw-Hill, 1996.

[4] Dluzewski, J.M. Large strain consolidation for elasto-plastic soils, NUMGE98, edited by A. Cividini, Udine, Italy 1998; 473-482.

[5] Highes, TJR. The Finite Element Method, Linear Static and Dynamic Finite Element Analysis, PRENTICE-HALL, Inc., 1987.

[6] Kos, J., Kuklík, P. and Šejnoha M. Laboratory measurements of material parameters of clayey soils, in preparation.

[7] Krejčí, T., Nový, T., Sehnoutek, L. and Šejnoha, J. Structure - subsoil interaction in view of transport processes in porous media, CTU Reports, Prague 2001; 5:1-81. 
[8] Kuklík, P., et. al. Verification of CAM clay model in description of consolidation, SSCC98, Durban, South Africa, 1998; 281-285.

[9] Kuklík, P., Mareš, J. and Šejnoha, M. Evaluation of the modified cam clay model with reference to isotropic consolidation, CTU Reports, Prague 1999; 3:47-54.

[10] Kvasnička, V. Augmented simulated annealing adaption of feed-forward neural networks, Neural Network World, 1994; 3:67-80.

[11] Lewis, R. W., Schrefler, B. A. The finite element method in static and dynamic deformation and consolidation of porous media, Wiley, Chichester, NY, 1998.

[12] Matouš K., Lepš M., Zeman J. and Šejnoha M. Applying genetic algorithms to selected topics commonly encountered in engineering practice, Comput. Methods Appl. Mech. Engrg., 2000; 119:1600-1620.

[13] Michalewicz Z. Genetic Algorithms + Data Structures = Evolution programs, Springer-Verlag, 1992. 